\title{
Severity of Chest Imaging is Correlated with Risk of Acute Neuroimaging Findings among Patients with COVID-19
}

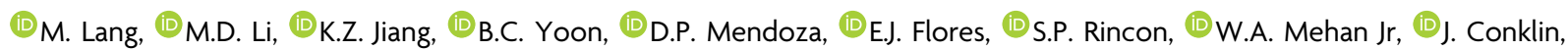

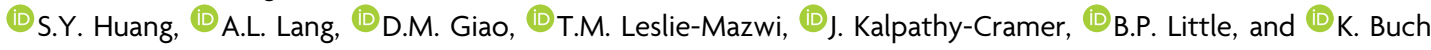

\begin{abstract}
BACKGROUND AND PURPOSE: Severe respiratory distress in patients with COVID-19 has been associated with higher rate of neurologic manifestations. Our aim was to investigate whether the severity of chest imaging findings among patients with coronavirus disease 2019 (COVID-19) correlates with the risk of acute neuroimaging findings.
\end{abstract}

MATERIALS AND METHODS: This retrospective study included all patients with COVID-19 who received care at our hospital
between March 3, 2020, and May 6, 2020, and underwent chest imaging within 10 days of neuroimaging. Chest radiographs were
assessed using a previously validated automated neural network algorithm for COVID-19 (Pulmonary X-ray Severity score). Chest
CTs were graded using a Chest CT Severity scoring system based on involvement of each lobe. Associations between chest imaging
severity scores and acute neuroimaging findings were assessed using multivariable logistic regression.

RESULTS: Twenty-four of 93 patients (26\%) included in the study had positive acute neuroimaging findings, including intracranial hemorrhage $(n=7)$, infarction $(n=7)$, leukoencephalopathy $(n=6)$, or a combination of findings $(n=4)$. The average length of hospitalization, prevalence of intensive care unit admission, and proportion of patients requiring intubation were significantly greater in patients with acute neuroimaging findings than in patients without them $(P<.05$ for all). Compared with patients without acute neuroimaging findings, patients with acute neuroimaging findings had significantly higher mean Pulmonary X-ray Severity scores (5.0 [SD, 2.9] versus 9.2 [SD, 3.4], $P<.001$ ) and mean Chest CT Severity scores (9.0 [SD, 5.1] versus 12.1 [SD, 5.0], $P=.041)$. The pulmonary $x$-ray severity score was a significant predictor of acute neuroimaging findings in patients with COVID-19.

CONCLUSIONS: Patients with COVID-19 and acute neuroimaging findings had more severe findings on chest imaging on both radiographs and CT compared with patients with COVID-19 without acute neuroimaging findings. The severity of findings on chest radiography was a strong predictor of acute neuroimaging findings in patients with COVID-19.

ABBREVIATIONS: $C C S=$ Chest CT Severity; COVID-19 = coronavirus disease 2019; ICU = intensive care unit; PXS = Pulmonary X-ray Severity; ROC = receiver operating characteristic; SARS-CoV-2 = Severe Acute Respiratory Syndrome coronavirus 2

$\mathbf{T}$ he Severe Acute Respiratory Syndrome coronavirus 2 (SARS-CoV-2), which causes coronavirus disease 2019 (COVID-19), has now infected $>40$ million people worldwide, with $>1$ million deaths reported by the end of October $2020{ }^{1}$

Received November 8, 2020; accepted after revision December 11.

From the Departments of Radiology (M.L., M.D.L., B.C.Y., D.P.M., E.J.F., S.P.R, W.A.M., J.C., S.Y.H., J.K.-C., B.P.L., K.B.), Anesthesia, Critical Care, and Pain Medicine (A.L.L.), Neurosurgery and Neurology (T.M.L.-M.), and Athinoula A. Martinos Center for Biomedical Imaging (J.C., S.Y.H., J.K.-C.), Massachusetts General Hospital, Harvard Medical School, Boston, Massachusetts; School of Medicine (K.Z.J.), Baylor College of Medicine, Houston, Texas; and Harvard Medical School (D.M.G.), Boston, Massachusetts.

B.P. Little and K. Buch are equal co-senior authors.

This study was supported by sundry funds to J. Kalpathy-Cramer. This research was carried out, in whole or in part, at the Athinoula A. Martinos Center for Biomedical Imaging at the Massachusetts General Hospital, using resources provided by the Center for Functional Neuroimaging Technologies, P41EB015896, a P41 Biotechnology Resource Grant supported by the National Institute of Biomedical Imaging and Bioengineering, National Institutes of Health. GPU computing resources were provided by the MGH and BWH Center for Clinical Data Science.
While COVID-19 is well-known for its pulmonary manifestations, it has been shown to involve other organs, including the heart, kidneys, liver, and central nervous system. ${ }^{2-5}$ This involvement is consistent with viral entry through the angiotensin-converting enzyme 2 receptor, which is abundantly expressed on vascular endothelial cells of the lungs but also in other organs, including the central nervous system, heart, kidneys, intestines, and muscles. ${ }^{6}$ Reported neurologic manifestations of COVID-19 infection include ischemic and hemorrhage stroke, encephalitis, and leukoencephalopathy. ${ }^{7-12}$

Please address correspondence to Min Lang, MD, MSc, Department of Radiology, Massachusetts General Hospital, 55 Fruit St, Boston, MA 02114; e-mail: mlang@mgh.harvard.edu

- Indicates open access to non-subscribers at www.ajnr.org

http://dx.doi.org/10.3174/ajnr.A7032 
Table 1: Summary of patient characteristics, clinical data, and indication for neuroimaging

\begin{tabular}{lccc}
\hline \multicolumn{1}{c}{ Demographics } & $\begin{array}{c}\text { With Neuroimaging } \\
\text { Findings }(\boldsymbol{n}=\mathbf{2 4})\end{array}$ & $\begin{array}{c}\text { Without Neuroimaging } \\
\text { Findings }(\boldsymbol{n}=69)\end{array}$ & $\begin{array}{c}\boldsymbol{P} \\
\text { Value }\end{array}$ \\
\hline Age (mean) [SD] (yr) & $63[\mathrm{SD}, 16]$ & $66[\mathrm{SD}, 16]$ & .396 \\
Female sex (No.) (\%) & $8(33)$ & $23(33)$ & 1.000 \\
Clinical data & & & \\
$\quad$ Length of stay (mean) [SD] (day) & $31[\mathrm{SD}, 12]$ & $23[\mathrm{SD}, 15]$ & .021 \\
ICU admission (No.) (\%) & $22(92)$ & $46(67)$ & .035 \\
Intubation (No.) (\%) & $21(88)$ & $36(52)$ & .005 \\
Death (No.) (\%) & $5(21)$ & $12(17)$ & .945 \\
Indication for neuroimaging & & & .089 \\
Altered mental status & $13(54)$ & $42(61)$ & \\
Concern for stroke & $10(42)$ & $14(20)$ & \\
Trauma & $0(0)$ & $10(15)$ & \\
Seizure & $1(4)$ & $1(3)$ & \\
Headache & $0(0)$ & $2(1)$ & \\
\hline
\end{tabular}

findings is correlated with the risk of positive neuroimaging findings in patients admitted for COVID-19 infection.

\section{MATERIALS AND METHODS \\ Study Cohort}

This retrospective study was approved by Massachusetts General Hospital institutional review board of our institution with a waiver of informed consent, and patient privacy was ensured in compliance with the Health Insurance Portability and Accountability Act. Between March 3, 2020, and May 6, 2020, a total of 641 patients received medical care at our quaternary care aca-
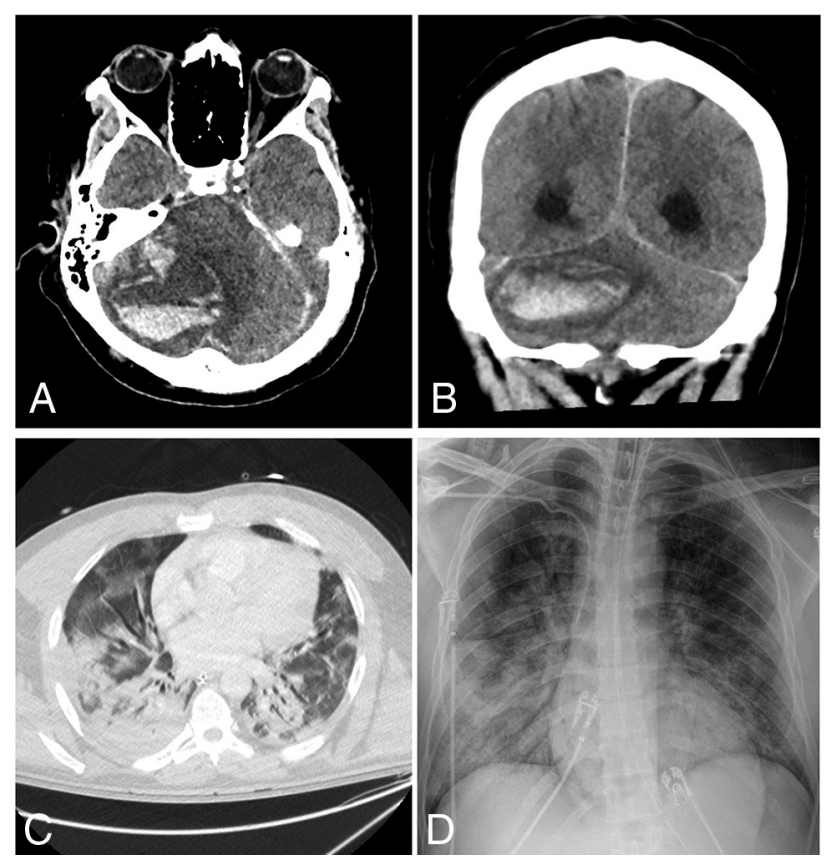

FIG 1. A 42-year-old man presented with hypoxemic respiratory failure. Non-contrast-enhanced axial $(A)$ and coronal $(B) C T$ head images demonstrate a new, large, right cerebellar intraparenchymal mixedattenuation hematoma suggestive of hyperacute or active hemorrhage. $C$, Chest CT of the patient demonstrates bilateral consolidation and ground-glass opacities involving all lobes, with a CCS score of 18. $D$, Portable chest radiograph demonstrates bilateral patchy air space opacities with a PXS score of 10.3.

It has been previously reported that patients with COVID-19 and severe respiratory distress have a relatively high rate of neurologic involvement. ${ }^{13}$ Furthermore, patients with COVID-19 and neurologic symptoms have been shown to have poorer outcomes than those without them. ${ }^{14}$ Thus, it would be important to identify clinical or imaging features in patients with COVID-19 that may help predict an increased risk of neurologic injury. Severity of disease on chest imaging is a strong predictor of clinical outcome and risk of complications. ${ }^{15-17}$ The objective of our study was to identify whether the severity of chest imaging demic medical center for COVID-19. Patients were included if they tested positive for SARS-CoV-2 infection diagnosed via real-time polymerase chain reaction, underwent $\mathrm{CT}$ and/or MR imaging for the evaluation of neurologic symptoms, and underwent chest radiography and/or CT within 10 days of neuroimaging for pulmonary symptoms. Patients were excluded if their neuroimaging was affected by substantial artifacts that precluded diagnostic assessment.

\section{Clinical Data}

Basic demographic information, including age and sex, as well as indications for neuroimaging were obtained from the electronic medical record for each patient. Clinical data, including length of hospital stay, admission to intensive care unit, intubation status, and death, were obtained for each patient.

\section{Imaging Technique}

All neuroimaging CT examinations were obtained on 64-section multidetector row CT scanners, either a Revolution CT (GE Healthcare) or a Somatom Force CT (Siemens) scanner. For noncontrast-enhanced head CTs, the studies were axially acquired (120 kV/auto-milliampere-seconds) at 5- and 1.25-mm-thick slices, from the level of the skull base to the vertex. For MR brain imaging, sequences performed included axial DWI, axial T2 FLAIR, axial T2WI, axial SWI, and sagittal T1WI. Intravenous contrast was used in a subset of studies.

All patients included in the study underwent portable chest radiography within 10 days before neuroimaging. The chest radiograph with the highest severity (highest Pulmonary X-ray Severity [PXS] score) within 10 days before neuroimaging was used for analysis (a mean of 3.3 [SD, 3.3] days for patients with acute neuroimaging findings versus a mean of 1.8 [SD, 3.7] days for patients without acute neuroimaging findings, $P=.18$ ).

Chest CT was performed in a subset of patients during admission and within 10 days before neuroimaging (a mean of 2.2 [SD, 2.9] days for patients with acute neuroimaging findings versus a mean of 1.5 [SD , 2.6] days for patients without acute neuroimaging findings, $P=.40$ ). The images were obtained with the patient in the supine position using one of the following CT scanners: Discovery CT750 HD (GE Healthcare), Revolution Frontier (GE 

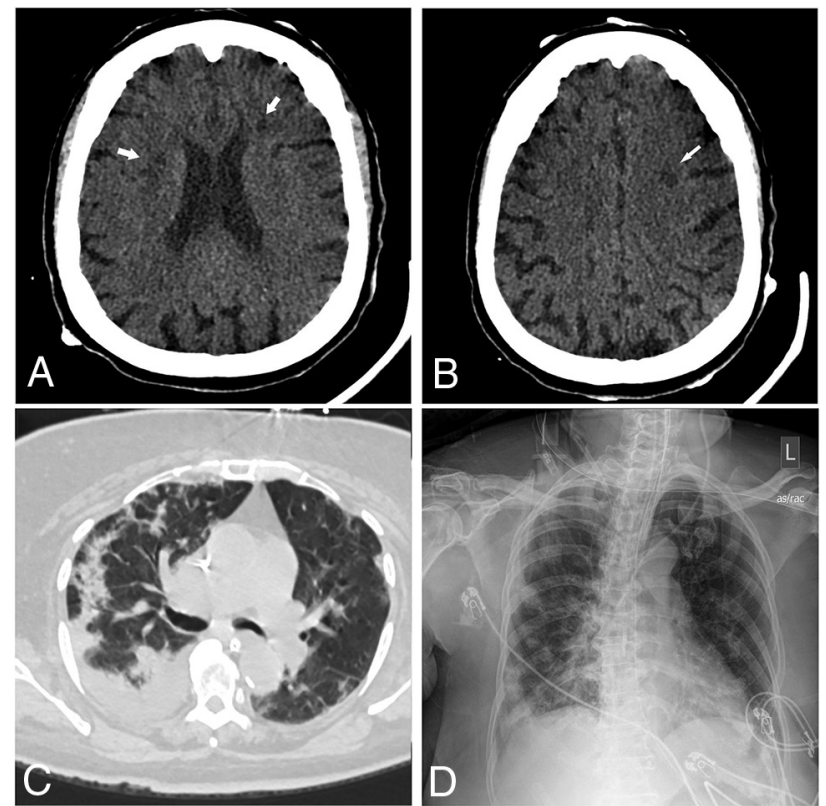

FIG 2. An 85-year-old man presented with increasing shortness of breath. Non-contrast-enhanced axial CT of the head demonstrates multiple new foci of hypoattenuation within the bilateral frontal corona radiata (arrows on $A$ ) and left centrum semiovale (arrow on B), in keeping with acute infarcts. $C$, Chest CT of the patient shows diffuse bilateral, right greater-than-left, predominantly consolidation and ground-glass opacities as well as bilateral pleural effusions. The CCS score was calculated as 13. D, Portable chest radiograph demonstrates bilateral patchy opacities with a PXS score of 10.6.

Healthcare), Somatom Definition Flash (Siemens), Somatom Definition AS (Siemens), Somatom Force (Siemens), and Aquilion ONE (Toshiba). The scanning parameters were the following: tube voltage $=140 \mathrm{kV}$ (peak) (plus $80 \mathrm{kVp}$ for dual energy), matrix $=512 \times 512$, section thickness $=1.25 \mathrm{~mm}, \mathrm{FOV}=$ $440 \times 440 \mathrm{~mm}$.

\section{Image Evaluation}

All neuroimaging examinations included in this study were reviewed by 2 fellowship-trained neuroradiologists, and the presence of intracranial hemorrhage, acute or subacute infarction, and/or leukoencephalopathy was recorded.

Portable chest radiographs for each patient were assessed using a previously validated convolutional Siamese neural network-based approach for automated assessment of COVID-19 lung disease severity, called the PXS score. ${ }^{16,18}$ Briefly, this machine learning model takes pixel-level image data from frontal chest radiographs as input and outputs a quantitative score for parenchymal lung disease severity. This score has previously been shown to correlate with manual radiologic assessments of COVID-19 radiographic severity as well as clinical outcome. A PXS score of $\leq 2.5$ indicates no or minimal disease; $>2.5$ and $\leq 5.0$, mild disease; $>5.0$ and $\leq 9.0$, moderate disease; and $>9.0$, severe disease. ${ }^{16,18}$

Chest CT severity was assessed by 3 fellowship-trained thoracic radiologists on the basis of a previously published grading system, the Chest CT Severity (CCS) score. ${ }^{19}$ Briefly, the 5 lung lobes were assessed individually for the degree of involvement by
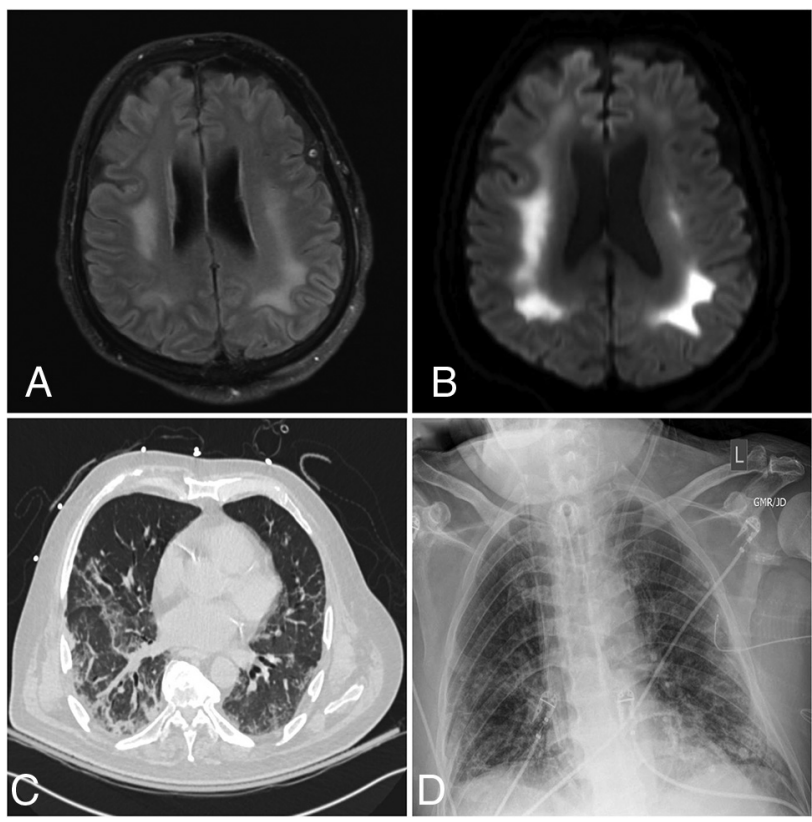

FIG 3. A 64-year-old man presented with fever and shortness of breath. Axial FLAIR $(A)$ and diffusion-weighted $(B)$ images demonstrate extensive symmetric confluent T2/FLAIR signal abnormality and restricted diffusion involving the corona radiata bilaterally. C, Chest CT shows bilateral peripheral ground-glass opacities and a small amount of consolidation involving all lobes, with a CCS score of 15. D, Portable chest radiograph demonstrates bilateral lower-zone-predominant peripheral opacities with a PXS score of 8.8 .

Table 2: Distribution of neuroimaging findings and RSNA categories

\begin{tabular}{cc}
\hline With & Without \\
Neuroimaging & Neuroimaging \\
Findings $(n=24)$ & Findings \\
\hline
\end{tabular}

\begin{tabular}{lll}
\hline $\begin{array}{l}\text { Acute neuroimaging } \\
\text { findings }\end{array}$ & \\
Infarction & $7(29)$ & NA \\
Hemorrhage & $7(29)$ & NA \\
Leukoencephalopathy & $6(25)$ & NA \\
Combination & $4(17)$ & NA \\
Chest CT RSNA & $n=14$ & $n=66$ \\
category & & \\
Typical & $9(64)$ & $34(52)$ \\
Indeterminate & $1(7)$ & $18(27)$ \\
Atypical & $4(29)$ & $10(15)$ \\
Negative & $0(0)$ & $4(6)$ \\
\hline
\end{tabular}

Note:-NA indicates not applicable; RSNA, Radiological Society of North America.

ground-glass opacities and consolidation. A score of 0 indicates no involvement; 1 , minimal involvement (1\%-25\%); 2 , mild involvement $(26 \%-50 \%)$; 3 , moderate involvement $(51 \%-75 \%)$; and 4 , severe involvement $(76 \%-100 \%)$. The summation of the individual lobe scores yields the total CCS score.

\section{Statistical Analysis}

Statistical analysis was performed using GraphPad Prism software (GraphPad Software) and R Studio (http://rstudio.org/ download/desktop). Continuous data were presented as means 


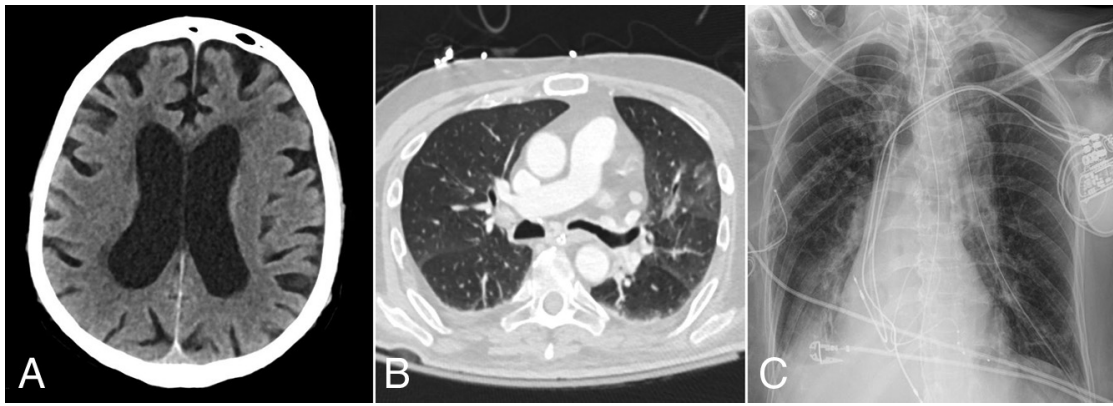

FIG 4. A 67-year-old woman presented with hypoxemic respiratory failure. Axial non-contrastenhanced head CT image (A) shows no acute findings. $B$, Chest CT image of the patient demonstrates bilateral peripherally distributed ground-glass opacities involving 4 of the 5 lobes with a CCS score of 8. D, Portable chest radiograph demonstrates bibasilar patchy opacities with a PXS score of 4.4 .
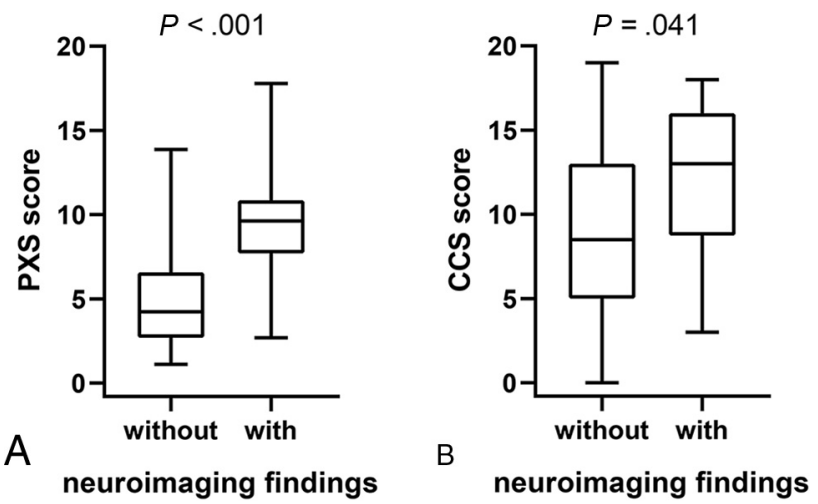

FIG 5. A, Comparison of portable chest radiograph severity (PXS) score between patients with and without acute neuroimaging findings. B, Comparison of the CCS score between patients with and without acute neuroimaging findings.

[SD] and were analyzed by a 2-tailed $t$ test; categoric data were presented as frequency (\%) and analyzed with a $\chi^{2}$ test. Associations between the lung disease severity scores (PXS and CCS) and neuroimaging findings were examined using multivariable logistic regression. Models were risk-adjusted for age, sex, the need for intubation, and intensive care unit (ICU) admission. The R Studio package pROC (https://www.rdocumentation.org/ packages/pROC/versions/1.16.2) was used to generate receiver operating characteristic (ROC) curves and to calculate the area under the ROC curve. ${ }^{20}$ We computed 95\% confidence intervals with 2000 stratified bootstrap replicates. Statistical significance was set at $P<.05$.

\section{RESULTS}

A total of 93 patients met the inclusion criteria for the study. Twenty-four (26\%) patients had acute neuroimaging findings, whereas $69(74 \%)$ patients did not. The proportion of patients who underwent MR imaging or CT was not significantly different between patients without acute neuroimaging findings (58 underwent head CT and 11 underwent brain MR imaging) and patients with acute neuroimaging findings (18 underwent head CT and 6 underwent brain MR imaging; $P=.36$ ). The mean age of patients without acute neuroimaging findings was $66[S D, 16]$ years of age, $33 \%$ of whom were women. The mean age for patients with acute neuroimaging findings was 63 [SD, 16] years, 33\% of whom were women as well. The most common indication for neuroimaging was altered mental status and concern for stroke for patients with and without neuroimaging findings (Table 1).

The average length of hospital stay was significantly longer in patients with acute neuroimaging findings (31 [SD , 12] days versus 23 [SD, 15] days, $P=.021$; Table 1 ). In addition, the prevalence of ICU admission and intubation was significantly higher for patients with acute neuroimaging findings than for patients without acute neuroimaging findings ( $92 \%$ versus $67 \%, P=.04 \%$, and $88 \%$ versus $52 \%, P=.005$, respectively; Table 1$)$. The mortality rate, however, was not significantly different between patients without acute neuroimaging findings (17\%) and patients with acute neuroimaging findings $(21 \%, P=.945)$.

Of the 24 patients with acute neuroimaging findings, 7 patients were found to have intracranial hemorrhage (Fig 1), 7 patients were found to have acute infarction (Fig 2), 6 patients were found to have leukoencephalopathy (Fig 3), 3 patients had intracranial hemorrhage and infarction, and 1 patient had intracranial hemorrhage, infarction, and leukoencephalopathy (Table $2)$. Sixty-nine patients did not have acute neuroimaging findings (Fig 4). There was no significant difference $(P=.225)$ between the distribution of Radiological Society of North America categories of chest CT findings between patients without acute neuroimaging findings $(n=66)$ and those with acute neuroimaging findings $(n=14)$, with the "typical" category being the most prevalent in both groups (Table 2). ${ }^{21}$

The mean PXS score was significantly greater in patients with acute neuroimaging findings $(n=23,9.2[\mathrm{SD}, 3.4])$ than in patients without acute neuroimaging findings $(n=64$, 5.0 [SD, 2.9], $P<.001$; Fig 5A). The PXS score was a significant predictor of positive acute neuroimaging findings in patients with COVID-19 after adjusting for age, sex, ICU admission, and intubation status (odds ratio $=1.45, P<.001$; Fig 6). The mean CCS score was also significantly greater in patients with acute neuroimaging findings $(n=14,12.1$ [SD, 5.0]) than in patients without acute neuroimaging findings $(n=64,9.0$ [SD, 5.1], $P=.041$; Fig 5B). However, after we adjusted for age, sex, ICU admission, and intubation status, the CCS score was not a significant predictor of positive acute neuroimaging findings in patients with COVID-19 (odds ratio $=1.08, P=.308$; Fig 6 ).

The area under the ROC curve using the PXS score to classify acute neuroimaging findings was 0.83 (95\% CI, 0.72-0.93; Fig $7 A$ ). The optimal threshold PXS score was 7.55 , which corresponded to a sensitivity of $83 \%$, specificity of $81 \%$, positive likelihood ratio of 4.7 , and negative likelihood ratio of 0.2 . By means of this threshold, meaning scores of $\geq 7.6$ were labeled as positive 
for acute neuroimaging findings and scores of $\leq 7.5$ were labeled as negative for acute neuroimaging findings, $77 \%$ of cases in our study would be correctly classified.

The area under the curve using the CCS score to classify acute neuroimaging findings was 0.67 (95\% CI, 0.50-0.84; Fig $7 B$ ). The optimal threshold CCS score was 12.5 . This threshold corresponded to a sensitivity of $64 \%$, specificity of $73 \%$, positive likelihood ratio of 2.4 , and negative likelihood ratio of 0.5 . By means of this threshold, meaning scores of $\geq 13$ were labeled as positive for acute neuroimaging findings and scores of $\leq 12$ were labeled as negative for acute neuroimaging findings, $61 \%$ of cases in our study would be correctly classified.

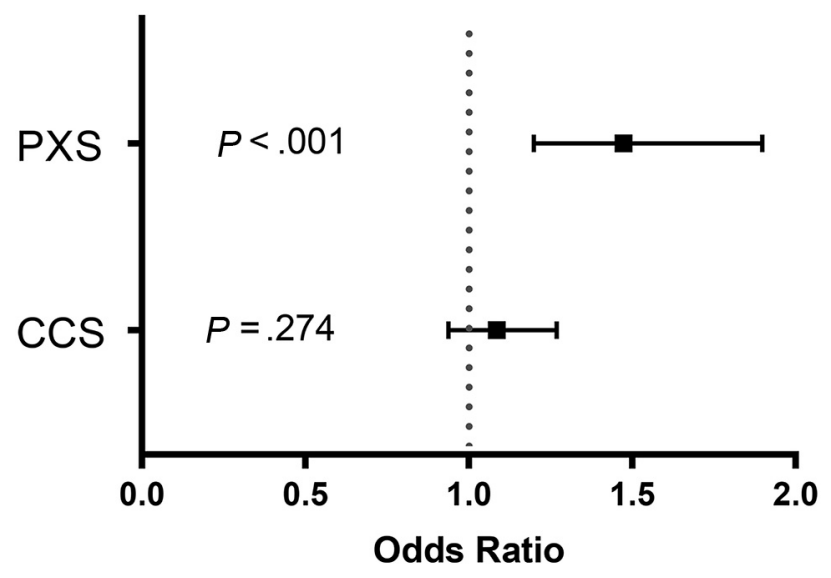

FIG 6. Odds ratio of the PXS score and the CCS score in association with the risk of acute neuroimaging findings. A multivariate logistic regression model was used after adjusting for age, sex, the need for intubation, and ICU admission status.
PXS Score

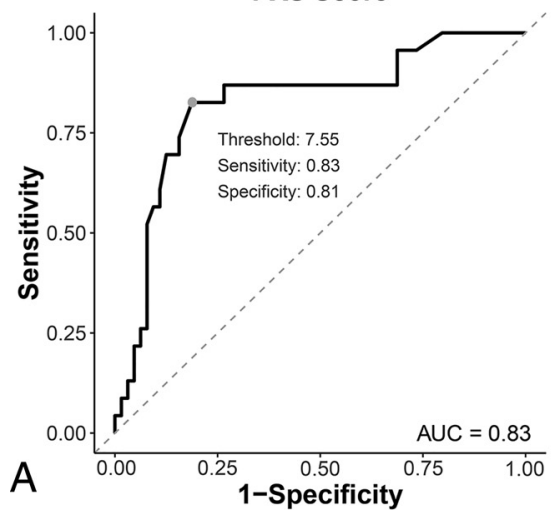

\section{CCS Score}

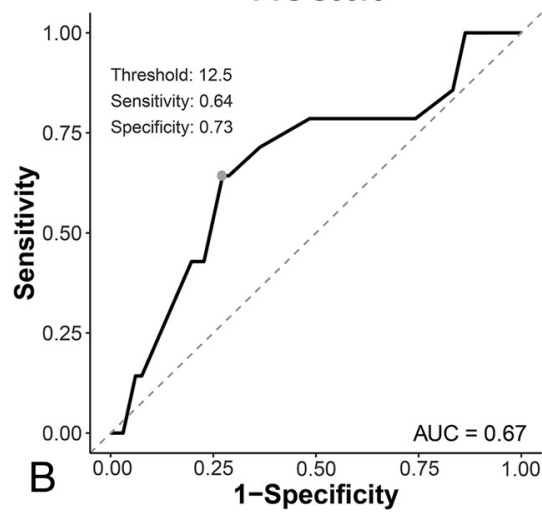

FIG 7. ROC curves of the PXS and CCS scores to classify acute neuroimaging findings in patients with COVID-19. A, The area under the ROC curve using the PXS score to classify acute neuroimaging findings was $0.83(95 \% \mathrm{Cl}, 0.72-0.93)$. The optimal threshold PXS score was 7.55, which corresponded to a sensitivity of $83 \%$, specificity of $81 \%$, positive likelihood ratio of 4.7 , and negative likelihood ratio of 0.2 . B, The area under the ROC curve using the CCS score to classify acute neuroimaging findings was $0.67(95 \% \mathrm{Cl}, 0.50-0.84)$. The optimal threshold CCS score was 12.5 , which corresponded to a sensitivity of $64 \%$, specificity of $73 \%$, positive likelihood ratio of 2.4 , and negative likelihood ratio of 0.5 .

\section{DISCUSSION}

A variety of neuroimaging findings have been reported in patients with COVID-19, including infarction, intracranial hemorrhage, leukoencephalopathy, and hemorrhagic encephalitis. ${ }^{8-11,14,22}$ The presence of acute-to-subacute neuroimaging findings in patients with COVID-19 is associated with a higher risk of ICU admission, intubation, and mortality. ${ }^{14}$ However, the risk factors for developing neurologic deficits in patients with COVID-19 are unclear. The results of this retrospective study showed that the severity of disease on chest radiographs and chest CT images was significantly greater among patients with COVID-19 with acute neuroimaging findings than in patients without acute neuroimaging findings, suggesting that more severe pulmonary manifestations are associated with a higher likelihood of neurologic injury.

Similar to prior reports, the presence of acute neuroimaging findings in our study was associated with an increased length of hospital stay, increased risk of requiring ICU admission, and risk of intubation. ${ }^{14,23}$ Acute ischemic and hemorrhagic strokes, the our study population, mortality was not significantly different between patients with acute neuroimaging findings and those significant difference.

Respiratory symptoms are the most common manifestations of COVID-19, and extensive research has been performed on chest imaging features of the disease, including using chest radiograph and chest CT severity as a prognostic tool for clinical outcome. $^{16,19,24-29}$ A study by Mao et $\mathrm{al}^{13}$ has also demonstrated high rates of neurologic symptoms in patients with COVID-19 and severe respiratory disease. This outcome may be due to shared mechanisms, including cytokine-related abnormal inflammatory response, coagulopathy, and microand macroangiopathy, which contribute to both chest and neuroimaging findings in patients with COVID$19 .^{28-34}$ Both chest and neuroimaging manifestations, however, are unlikely due to a single etiology but rather a combination of interconnected pathophysiologic processes. The greater severity of chest imaging findings may therefore indicate more severe systemwide pathology, in which case-heightened suspicion for acute neurologic injury is warranted. Consistent with this theory, PXS scores of patients with COVID-19 were found to be significantly associated with an increased risk of acute neuroimaging findings in our study. While a higher CCS score was associated with an increased risk 
of acute neuroimaging findings in the current study, this did not reach statistical significance. This may be related to the small number of patients who underwent chest CT relative to the number of patients who underwent chest radiographic assessment. Future investigation with a larger patient cohort is needed.

Three patients in the current study who had minimal-to-mild chest radiographic findings were found to have either infarction or intracranial hemorrhage. Similarly, 3 patients with mild disease on chest CT were found to have infarction, hemorrhage, and leukoencephalopathy on neuroimaging. The cause of neurologic injury in these patients may be better explained by direct neurotropism of the SARS-CoV-2 virus, which may occur through retrograde transmission through peripheral nerve terminals including the olfactory nerves, rather than widespread cytokine storm. ${ }^{35,36}$ Other proposed mechanisms of neurologic injury without severe chest imaging manifestations include silent hypoxia, metabolic disturbances, neuroinflammation, angiopathy, embolic phenomenon, or an autoimmune-mediated process. $^{37-40}$ While neurologic injury can occur in patients with mild pulmonary disease and with less evident chest imaging findings, the relative proportion of these patients is much smaller than those with more severe pulmonary findings.

Limitations of the current study include its retrospective nature and relatively small sample size from a single institution. The small number of patients also precluded further analysis of the risk of developing different types of neurologic injury, such as infarction, hemorrhage, or leukoencephalopathy. Furthermore, additional patient information including laboratory values and comorbidities was incomplete and therefore was not included in the study. Finally, most of these patients did not have additional imaging of other body parts, including the abdomen and pelvis, limiting correlation of findings in other organ systems.

\section{CONCLUSIONS}

Patients with COVID-19 and acute neuroimaging findings had greater disease severity on both chest radiography and CT than patients without acute neuroimaging findings. In our adjusted regression models, chest radiograph severity was a strong predictor of acute neuroimaging findings in patients with COVID-19, while the small number of patients may have underpowered the study to detect a significant correlation with chest CT. Therefore, a high severity of disease on chest imaging should raise suspicion for neurologic injury in patients with COVID-19. Early use of neuroimaging in these patients should be considered.

Disclosures: Matthew D. Li-UNRELATED: Grants/Grants Pending: Radiological Society of North America, Comments: M.D.L. reports funding from a Radiological Society of North America Research and Education Presidents Circle Research Resident Grant, outside of the submitted work.* Efren J. FloresUNRELATED: Grants/Grants Pending: National Cancer Institute Research Diversity Supplement, American College of Radiology Innovation Fund. William A. Mehan, Jr-UNRELATED: Consultancy: Kura Oncology, Comments: independent reviewer of imaging for a head and neck cancer trial; Employment: Massachusetts General Hospital; Expert Testimony: expert opinion for medicolegal cases involving neuroimaging. Susie Y. Huang_UNRELATED: Grants/Grants Pending: Siemens, Comments: research grant for clinical translation of fast brain MRI sequences*; Payment for Lectures Including Service on Speakers Bureaus: Siemens, Comments: payment for speaking on Connectome 2.0 at OHBM 2020. Jayashree KalpathyCramer-UNRELATED: Grants/Grants Pending: GE Healthcare, Genentech
Foundation*; Travel/Accommodations/Meeting Expenses Unrelated to Activities Listed: IBM. Brent P. Little—UNRELATED: Royalties: Elsevier, Comments: textbook royalties, chest radiology textbook, author/associate editor. *Money paid to the institution.

\section{REFERENCES}

1. Johns Hopkins University. COVID-19 Dashboard by the Center for Systems Science and Engineering (CSSE) at Johns Hopkins University (JHU). January 7, 2021. https://coronavirus.jhu.edu/map. html. Accessed November 4, 2020

2. Bhayana R, Som A, Li MD, et al. Abdominal imaging findings in COVID-19: preliminary observations. Radiology 2020;297:E207-15 CrossRef Medline

3. Puelles VG, Lütgehetmann M, Lindenmeyer MT, et al. Multiorgan and renal tropism of SARS-CoV-2. N Engl J Med 2020;383:590-92 CrossRef Medline

4. Zaim S, Chong JH, Sankaranarayanan V, et al. COVID-19 and multiorgan response. Curr Probl Cardiol 2020;45:100618 CrossRef Medline

5. Wang D, Hu B, Hu C, et al. Clinical characteristics of 138 hospitalized patients with 2019 novel coronavirus-infected pneumonia in Wuhan, China. JAMA 2020;323:1061-69 CrossRef Medline

6. Li MY, Li L, Zhang Y, et al. Expression of the SARS-CoV-2 cell receptor gene ACE2 in a wide variety of human tissues. Infect Dis Poverty 2020;9:45 CrossRef Medline

7. De Felice FG, Tovar-Moll F, Moll J, et al. Severe acute respiratory syndrome coronavirus 2 (SARS-CoV-2) and the central nervous system. Trends Neurosci 2020;43:355-57 CrossRef Medline

8. Poyiadji N, Shahin G, Noujaim D, et al. COVID-19-associated acute hemorrhagic necrotizing encephalopathy: imaging features. Radiology 2020;296:E119-20 CrossRef Medline

9. Ye M, Ren Y, Lv T. Encephalitis as a clinical manifestation of COVID-19. Brain Behav Immun 2020;88:945-46 CrossRef Medline

10. Moriguchi T, Harii N, Goto J, et al. A first case of meningitis/encephalitis associated with SARS-coronavirus-2. Int J Infect Dis 2020;94:55-58 CrossRef Medline

11. Lang M, Li MD, Buch K, et al. Risk of acute cerebrovascular events in patients with COVID-19 infection. AJNR Am J Neuroradiol 2020;41:E92-93 CrossRef Medline

12. Josephson SA, Kamel H. Neurology and COVID-19. JAMA 2020;324:1139-40 CrossRef Medline

13. Mao L, Jin H, Wang M, et al. Neurologic manifestations of hospitalized patients with coronavirus disease 2019 in Wuhan, China. JAMA Neurol 2020;77:683-90 CrossRef Medline

14. Jain R, Young M, Dogra S, et al. COVID-19 related neuroimaging findings: a signal of thromboembolic complications and a strong prognostic marker of poor patient outcome. J Neurol Sci 2020;414: 116923 CrossRef Medline

15. Feng Z, Yu Q, Yao S, et al. Early prediction of disease progression in COVID-19 pneumonia patients with chest CT and clinical characteristics. Nat Commun 2020;11:4968 CrossRef Medline

16. Li MD, Arun NT, Gidwani M, et al. Automated assessment and tracking of COVID-19 pulmonary disease severity on chest radiographs using convolutional Siamese neural networks. Radiology: Artificial Intelligence $2020 \mathrm{Jul}$ 22. [Epub ahead of print] CrossRef

17. Toussie D, Voutsinas N, Finkelstein M, et al. Clinical and chestradiography features determine patient outcomes in young and middle-aged adults with COVID-19. Radiology 2020;297:E197-206 CrossRef Medline

18. Li MD, Arun NT, Aggarwal M, et al. Improvement and multi-population generalizability of a deep learning-based chest radiograph severity score for COVID-19. medRxiv 2020. CrossRef. Accessed November 5, 2020

19. Steinberger S, Lin B, Bernheim A, et al. CT features of coronavirus disease (COVID-19) in 30 pediatric patients. AJR Am J Roentgenol 2020;215:1303-11 CrossRef Medline 
20. Robin X, Turck N, Hainard A, et al. pROC: an open-source package for $\mathbf{R}$ and $\mathrm{S}+$ to analyze and compare ROC curves. $B M C$ Bioinformatics 2011;12:77 CrossRef Medline

21. Simpson S, Kay FU, Abbara S, et al. Radiological Society of North America Expert Consensus Statement on Reporting Chest CT Findings Related to COVID-19: endorsed by the Society of Thoracic Radiology, the American College of Radiology, and Radiological Society of North America-secondary publication. $J$ Thorac Imaging 2020;35:219-27 CrossRef Medline

22. Lang $\mathrm{M}$, Buch $\mathrm{K}, \mathrm{Li} \mathrm{MD}$, et al. Leukoencephalopathy associated with severe COVID-19 infection: sequela of hypoxemia? AJNR Am J Neuroradiol 2020;41:1641-45 CrossRef Medline

23. Yoon $\mathrm{BC}$, Buch $\mathrm{K}$, Lang $\mathrm{M}$, et al. Clinical and neuroimaging correlation in patients with COVID-19. AJNR Am J Neuroradiol 2020; 41:1791-96 CrossRef Medline

24. Huang C, Wang Y, Li X, et al. Clinical features of patients infected with 2019 novel coronavirus in Wuhan. Lancet 2020;395:497-506 CrossRef Medline

25. Pan Y, Guan H, Zhou S, et al. Initial CT findings and temporal changes in patients with the novel coronavirus pneumonia (2019nCoV): a study of 63 patients in Wuhan, China. Eur Radiol 2020; 30:3306-09 CrossRef Medline

26. Shi $\mathrm{H}, \mathrm{Han} \mathrm{X}$, Jiang $\mathrm{N}$, et al. Radiological findings from 81 patients with COVID-19 pneumonia in Wuhan, China: a descriptive study. Lancet Infect Dis 2020;20:425-34 CrossRef Medline

27. Chung M, Bernheim A, Mei X, et al. CT imaging features of 2019 novel coronavirus (2019-nCoV). Radiology 2020;295:20207 CrossRef Medline

28. Lang M, Som A, Mendoza DP, et al. Hypoxaemia related to COVID-19: vascular and perfusion abnormalities on dual-energy CT. Lancet Infect Dis 2020;20:1365-66 CrossRef Medline

29. Lang M, Som A, Carey D, et al. Pulmonary vascular manifestations of COVID-19 pneumonia. Radiology: Cardiothoracic Imaging 2020; 2:e200277 CrossRef
30. Scholkmann F, Nicholls J. Pulmonary vascular pathology in COVID-19. N Engl J Med 2020;383:887-88 CrossRef Medline

31. Ackermann M, Verleden SE, Kuehnel M, et al. Pulmonary vascular endotheliitis, thrombosis, and angiogenesis in COVID-19. $N$ Engl J Med 2020;383:120-28 CrossRef Medline

32. Chan NC, Weitz JI. COVID-19 coagulopathy, thrombosis, and bleeding. Blood 2020;136:381-83 CrossRef Medline

33. Becker RC. COVID-19 update: COVID-19-associated coagulopathy. J Thromb Thrombolysis 2020;50:54-67 CrossRef Medline

34. McElvaney OJ, McEvoy NL, McElvaney OF, et al. Characterization of the inflammatory response to severe COVID-19 illness. $\mathrm{Am} \mathrm{J}$ Respir Crit Care Med 2020;202:812-21 CrossRef Medline

35. Mehraeen E, Behnezhad F, Amin Salehi M, et al. Olfactory and gustatory dysfunctions due to the coronavirus disease (COVID-19): a review of current evidence. Eur Arch Otorhinolaryngol 2020 Jun 17. [Epub ahead of print] CrossRef Medline

36. Conde Cardona G, Quintana Pajaro LD, Quintero Marzola ID, et al. Neurotropism of SARS-CoV 2: mechanisms and manifestations. J Neurol Sci 2020;412:116824 CrossRef Medline

37. Delorme C, Paccoud O, Kas A, et al; CoCo-Neurosciences study group and COVID SMIT PSL study group. Covid-19-related encephalopathy: a case series with brain FDG-positron-emission tomography/computed tomography findings. Eur J Neurol 2020;27: 2651-57 CrossRef Medline

38. Fontana IC, Bongarzone S, Gee A, et al. PET imaging as a tool for assessing COVID-19 brain changes. Trends Neurosci 2020;43:93538 CrossRef Medline

39. Wenting A, Gruters A, van Os Y, et al. COVID-19 neurological manifestations and underlying mechanisms: a scoping review. Front Psychiatry 2020;11:860 CrossRef Medline

40. Kreye J, Reincke SM, Pruss H. Do cross-reactive antibodies cause neuropathology in COVID-19? Nat Rev Immunol 2020;20:645-46 CrossRef Medline 